\title{
The Relationship between Diabetes-Related Complications and Obstructive Sleep Apnea in Type 2 Diabetes
}

\author{
Nantaporn Siwasaranond, ${ }^{1}$ Hataikarn Nimitphong ${ }^{D},{ }^{1}$ Areesa Manodpitipong, \\ Sunee Saetung, ${ }^{1}$ Naricha Chirakalwasan, ${ }^{2,3}$ Ammarin Thakkinstian ${ }^{D},{ }^{4}$ \\ and Sirimon Reutrakul $\mathbb{D}^{1,5}$ \\ ${ }^{1}$ Division of Endocrinology and Metabolism, Department of Medicine, Faculty of Medicine, Ramathibodi Hospital, \\ Bangkok, Thailand \\ ${ }^{2}$ Division of Pulmonary and Critical Care Medicine, Department of Medicine, Faculty of Medicine, Chulalongkorn University, \\ Bangkok, Thailand \\ ${ }^{3}$ Excellence Center for Sleep Disorders, King Chulalongkorn Memorial Hospital, Thai Red Cross Society, Bangkok, Thailand \\ ${ }^{4}$ Section for Clinical Epidemiology and Biostatistics, Faculty of Medicine, Ramathibodi Hospital, Bangkok, Thailand \\ ${ }^{5}$ Division of Endocrinology, Diabetes and Metabolism, Department of Medicine, University of Illinois at Chicago, Chicago, IL, USA
}

Correspondence should be addressed to Sirimon Reutrakul; sreutrak10800@gmail.com

Received 5 November 2017; Revised 21 December 2017; Accepted 14 January 2018; Published 7 March 2018

Academic Editor: Patrizio Tatti

Copyright (c) 2018 Nantaporn Siwasaranond et al. This is an open access article distributed under the Creative Commons Attribution License, which permits unrestricted use, distribution, and reproduction in any medium, provided the original work is properly cited.

\begin{abstract}
This study explored the relationship between obstructive sleep apnea (OSA) and the presence of any diabetes-related complications in type 2 diabetes and whether this was mediated by hypertension. Secondly, the relationship between OSA severity and estimated glomerular filtration rate (eGFR) was investigated. A total of 131 patients participated. OSA was diagnosed using a home monitor, and severity was measured by apnea-hypopnea index (AHI) and oxygen desaturation index (ODI). OSA was found in $75.6 \%$ of the participants, $40.5 \%$ with moderate-to-severe degree. Any diabetes-related complications (retinopathy, neuropathy, nephropathy, or coronary artery disease) were present in $55.5 \%$, and $70.2 \%$ of the participants had hypertension. Mediation analysis indicated that, compared to those with mild or no OSA, those with moderate-to-severe OSA were 3.05 times more likely to have any diabetesrelated complications and that this relationship was mediated by the presence of hypertension. After adjusting for confounders, ODI $(B=-0.036, p=0.041)$, but not AHI, was significantly associated with lower eGFR. In conclusion, moderate-to-severe OSA was related to the presence of any diabetes-related complications in type 2 diabetes, and the relationship was mediated by hypertension. The severity of intermittent hypoxia was associated with lower eGFR. Whether OSA treatment will delay or reduce diabetes-related complications should be investigated.
\end{abstract}

\section{Introduction}

Obstructive sleep apnea (OSA) is a common sleep disorder, characterized by repetitive episodes of upper airway closure or partial collapse during sleep, resulting in intermittent hypoxia and fragmented sleep. OSA is a recognized risk factor for insulin resistance and type 2 diabetes (T2DM), independently of body mass index (BMI) [1]. In well-designed laboratory experiments, intermittent hypoxia and fragmented sleep resulted in increased insulin resistance without an adequate compensatory insulin response, leading to glucose intolerance [2,3]. Furthermore, a recent meta-analysis of over 60,000 participants from nine prospective cohort studies revealed that OSA was associated with a $35 \%$ increase in the risk of developing T2DM [1]. While obesity is a well-known risk factor for OSA, there are additional risk factors such as abnormalities in craniofacial and upper airway structure, particularly in Asian populations [4]. Furthermore, muscular dystrophy or other neuromuscular diseases can contribute to obstructive events when presenting as pharyngeal weakness 
or bulbar involvement [5]. Untreated OSA may impair daytime function and is associated with an increased risk of psychiatric symptoms such as mood or anxiety disorders [6]. The estimated overall prevalence of OSA has substantially increased, and it is highly prevalent in patients with T2DM, between $58-86 \%$, depending on the study population $[7,8]$, particularly in very obese patients with T2DM [9]. In patients with T2DM, OSA severity has been shown to be associated with poorer glycemic control [10, 11]. Despite the strong association between OSA and glucose metabolism, the effects of continuous positive airway pressure (CPAP) treatment on glycemic control in those with T2DM have been mixed, with some showing favorable effects while others did not $[12,13]$. Baseline glycemic status, study design, CPAP compliance, and duration of use may all play a role in these differing results [1].

Poor glycemic control in the long term contributes to diabetic micro- and macrovascular complications [14, 15], resulting in increased morbidity and mortality. Recent data have suggested that the presence and severity of OSA may pose an additional risk factor for these complications [16-19]. Vasoconstriction and increased oxidative stress as a result of intermittent hypoxia can lead to endothelial dysfunction and microvascular impairment [20]. Additionally, activation of the renin-angiotensin-aldosterone system (RAAS) in OSA patients could facilitate the development and progression of diabetic kidney disease (DKD) [21]. In a meta-analysis of 7 studies, OSA was associated with a 59\% higher risk of DKD [16]. Moreover, a recent longitudinal cohort study of patients with T2DM, followed for a median of 43 months, found an independent association between OSA and the risk of progression to advanced diabetic retinopathy [18]. Thus, these early data highlight the role of OSA in diabetes-related complications.

It is well known that OSA, especially moderate-to-severe degree, is strongly associated with hypertension [22], and hypertensive patients with OSA are at a higher risk for adverse cardiovascular events [23]. Hypertension also plays a crucial role in diabetes-related complications; systolic blood pressure has been shown to independently and additively exert effects on micro- and macrovascular complications in T2DM patients, in addition to glycemic control [24]. It is likely that hypertension at least partly mediates the relationship between OSA and diabetes complications.

Therefore, the purpose of this study was to investigate the relationship between OSA and diabetes-related complications in T2DM patients and explore whether this relationship was mediated by hypertensive state. Mediation analysis was employed to assess the direct and indirect effect (through hypertension) of OSA on these complications. Secondly, it was explored whether OSA severity was an independent predictor of kidney function, as measured by estimated glomerular filtration rate (eGFR), in T2DM.

\section{Methods}

Nonpregnant adults with T2DM without previously diagnosed OSA being followed at three hospitals in Thailand were invited to participate. The participants were a part of previously described cohorts $[25,26]$. Exclusion criteria included those with a history of congestive heart failure or low ejection fraction, chronic obstructive pulmonary disease, end-stage renal disease (ESRD), severe chronic liver disease (such as cirrhosis), stroke, permanent pacemaker placement, or use of certain medications (opioids/narcotics, alphaadrenergic blockers, clonidine, methyldopa, and nitroglycerin), in order to obtain valid results from the OSA diagnostic method utilized (see below). All participants gave written informed consent. The protocol was approved by the Ethical Clearance Committee, Faculty of Medicine Ramathibodi Hospital, Bangkok (ID 11-60-95).

\subsection{Assessment of Baseline Characteristics, Glycemic Control,} and Diabetes Complications. Weight and neck circumference $(\mathrm{cm})$ were measured. Height, age, current medications (i.e., antidiabetic medications, statins, angiotensin-converting enzyme inhibitor (ACEI), or angiotensin receptor blocker (ARB)), and most recent hemoglobin Alc (HbAlc) values (within three months) were extracted from patient medical records. HbAlc is an index of glycemic control over the preceding 90 days. Body mass index (BMI) was calculated as weight $(\mathrm{kg}) /$ height $\left(\mathrm{m}^{2}\right)$. Hypertension was defined as having a clinical diagnosis of hypertension or receiving antihypertensive medication.

Diabetic complications were assessed by interviews and medical record reviews. A history of diabetic retinopathy was obtained from ophthalmologic examinations (within one year) and categorized into those with retinopathy versus no retinopathy. Diabetic kidney disease (DKD) was defined as an estimated glomerular filtration rate (eGFR) $<60 \mathrm{~mL} /$ $\min / 1.73 \mathrm{~m}^{2}$ (calculated using the Chronic Kidney Disease Epidemiology Collaboration equation) and/or presence of albuminuria $\geq 30 \mathrm{mg} / \mathrm{g}$ of creatinine or overt proteinuria, except in 3 patients for whom albuminuria was defined as the presence of albuminuria $\geq 30 \mathrm{mg}$. Peripheral neuropathy was defined as the presence of symptoms of peripheral neuropathy such as pain or burning sensation. Presence of coronary artery disease was obtained from an interview and confirmed by a medical record review. Presence of any diabetes-related complications was defined as having one or more complications.

2.2. Assessment of OSA. OSA was diagnosed utilizing an in-home overnight monitoring device, WatchPAT 200 (Itamar Medical, Caesarea, Israel). This is a noninvasive home sleep test device for detecting peripheral arterial tone (PAT) by a signal that measures the arterial pulsatile volume and reflects sympathetic nervous system augmentation due to desaturation during sleep. It has been validated against polysomnography [27]. Using specific signal patterns, a clinically validated algorithm provides indices used for determining the degree of sleep apnea. OSA was diagnosed if the apnea-hypopnea index (AHI) was $\geq 5$. Moderate-to-severe OSA was defined as $\mathrm{AHI} \geq 15$. Oxygen desaturation index (ODI) is the number of times per hour of sleep that the oxygen level decreases by at least $3 \%$ from baseline. 
2.3. Statistical Analysis. Data are presented as the mean \pm standard deviation (SD), median with interquartile range (IQR), or frequency with percent. Mann-Whitney $U$ test and independent $t$-test were used to compare continuous variables between those with and without any diabetesrelated complications. The chi-square test was used to compare categorical variables.

To investigate whether OSA had direct effects on diabetic complications or whether this was indirectly mediated by the presence of hypertension, mediation analysis was performed. A mediation analysis is a standard statistical method which attempts to identify whether the relationship between an independent variable and an outcome variable is mediated by a third, or "mediator," variable [28]. In this study, the presence of moderate-to-severe OSA (AHI $\geq 15)$ was an independent variable, hypertension was a mediator, and any diabetic complications were an outcome variable. First, a mediation model was constructed by fitting moderate-tosevere OSA as an independent variable on hypertension, adjusting for covariables including age, sex, BMI, diabetes duration, $\mathrm{HbA1c}$, and statin use. Second, an outcome model was constructed by fitting any diabetes-related complications on hypertension and moderate-to-severe OSA, adjusting for covariables as previously described. Only significant covariables were kept in each mediation and outcome model. An indirect effect of moderate-to-severe OSA on any diabetesrelated complications through hypertension was then estimated by multiplying coefficients of OSA in the mediation model with the coefficient of hypertension in the outcome model. A bootstrap of 1000 replications was then applied to the estimated indirect effect and its 95\% confidence interval (CI). These analyses were performed using a generalized structural equation model, with a family of Bernoulli distributions and logit link function for both mediation and outcome models. All analyses were done using Stata statistical software version 14.0.

To determine if OSA severity was associated with eGFR, the following analyses were performed. Univariate linear regression analysis was used to determine the association between baseline characteristics, $\mathrm{HbAlc}$, hypertension, ACEI/ARB use, sleep parameters (AHI and ODI), and eGFR. Stepwise backward regression analysis, adjusting for age, $\mathrm{BMI}, \mathrm{HbAlc}$, diabetes duration, hypertension, ACEI/ARB use, and statin use, was utilized to determine if AHI or ODI was an independent predictor of eGFR. For these analyses, natural logarithm-transformed values ( $\mathrm{Ln}$ ) of $\mathrm{HbAlc}$, diabetes duration, eGFR, AHI, and ODI were used, since they were not normally distributed; $p$ values $<0.05$ were considered statistically significant.

\section{Results}

A total of 131 patients participated in the study. Their demographic and glycemic characteristics, diabetes-related complications, and sleep parameters are shown in Table 1. The mean age was 52.6 years, $55 \%$ were female, and median HbAlc was $7.4 \%$. Forty-eight $(37.2 \%)$ participants had DKD, 25 (19.5\%) had retinopathy, 30 (23.3\%) reported neuropathy symptoms, and overall 71 (55.5\%) participants had
TABLE 1: Baseline characteristics of all participants.

\begin{tabular}{lc}
\hline & $n=131$ \\
\hline Demographic and glycemic characteristics & \\
Age (years) & $52.6(11.5)$ \\
Sex (female) & $72(55.0 \%)$ \\
BMI $\left(\mathrm{kg} / \mathrm{m}^{2}\right)$ & $28.8(5.1)$ \\
Neck circumference $(\mathrm{cm})$ & $37.7(3.9)$ \\
Diabetes duration (years) & $10.3(8.7)$ \\
HbA1c $(\%)$ & $7.43(6.68,8.74)$ \\
Hypertension & $92(70.2 \%)$ \\
ACEI or ARB use & $72(55.0 \%)$ \\
Statin use & $104(79.4 \%)$ \\
eGFR (mL/min/1.73 m $\left.{ }^{2}\right)$ & $94.5(79.6,104.6)$ \\
Serum creatinine $(\mathrm{mg} / \mathrm{dL})$ & $0.87(0.37)$ \\
Diabetic complications & \\
Diabetic nephropathy & $48(37.2 \%)$ \\
Diabetic retinopathy & $25(19.5 \%)$ \\
Neuropathy & $30(23.3 \%)$ \\
Coronary artery disease & $7(5.3 \%)$ \\
Any complications & $71(55.5 \%)$ \\
Sleep parameters & \\
AHI & $10.9(5.2,21.3)$ \\
ODI & $6.6(2.0,14.0)$ \\
OSA diagnosis (AHI $\geq 5)$ & $99(75.6 \%)$ \\
AHI $\geq 15$ & $53(40.5 \%)$ \\
\hline Datare &
\end{tabular}

Data are presented as mean (SD), median (IQR), or number (\%). ACEI: angiotensin-converting enzyme inhibitor; ARB: angiotensin receptor blocker; AHI: apnea hypopnea index; eGFR: estimated glomerular filtration rate; HbAlc: hemoglobin Alc; ODI: oxygen desaturation index.

at least one diabetes-related complication. Median (IQR) eGFR was $94.5 \mathrm{~mL} / \mathrm{min} / 1.73 \mathrm{~m}^{2}(79.6,104.6)$. Over threequarters of the participants had OSA, and $40.5 \%$ had moderate-to-severe OSA, with an overall median AHI (IQR) of $10.9(5.2,21.3)$.

3.1. OSA, Diabetes-Related Complications, and Hypertension. When categorizing the participants into those with or without any diabetes-related complications, it was found that those with any complications had poorer glycemic control and were more likely to have hypertension and be receiving treatment with ACEI/ARB (Table 2). In addition, they had more severe OSA, as indicated by higher median AHI and ODI, and were more likely to be diagnosed with OSA than those without complications. More of those with any complications had moderate-to-severe OSA than those without complications ( $45.1 \%$ versus $35.1 \%$ ), although this did not reach statistically significant levels.

Because those with hypertension were more likely to have diabetes-related complications and it is known that OSA can lead to hypertension, we explored further by categorizing participants according to their diagnosis of hypertension (Table 2). This revealed that those with hypertension were older, had higher BMI, were more likely to be using ACEI/ARB, and had more severe OSA 
TABLE 2: Comparisons between participants without and with any diabetes-related complications and those with and without hypertension.

\begin{tabular}{|c|c|c|c|c|c|c|}
\hline & $\begin{array}{c}\text { No complications } \\
\qquad(n=57)\end{array}$ & $\begin{array}{c}\text { Presence of any diabetes } \\
\text { complications }(n=71)\end{array}$ & $p$ value & $\begin{array}{l}\text { Normotensive } \\
\quad(n=39)\end{array}$ & $\begin{array}{l}\text { Hypertensive } \\
\quad(n=92)\end{array}$ & $p$ value \\
\hline \multicolumn{7}{|c|}{ Demographic and glycemic characteristics } \\
\hline Age (years) & $51.2(10.9)$ & $53.8(11.5)$ & 0.189 & $47.3(13.0)$ & $54.8(10.0)$ & 0.001 \\
\hline Sex (female) & $32(56.1 \%)$ & $39(54.9 \%)$ & 0.891 & $25(64.1 \%)$ & $47(51.1 \%)$ & 0.171 \\
\hline BMI $\left(\mathrm{kg} / \mathrm{m}^{2}\right)$ & $27.8(5.1)$ & $29.3(4.9)$ & 0.100 & $27.2(4.7)$ & $29.4(5.1)$ & 0.023 \\
\hline Diabetes duration (years) & $9.4(9.3)$ & $11.2(8.5)$ & 0.250 & $8.5(8.4)$ & $11.0(8.9)$ & 0.141 \\
\hline HbAlc (\%) & $7.2(6.6,7.8)$ & $7.9(6.7,9.5)$ & 0.023 & $7.8(6.6,9.8)$ & $7.3(6.7,8.6)$ & 0.350 \\
\hline Hypertension & $33(57.9 \%)$ & $57(80.3 \%)$ & 0.006 & - & - & - \\
\hline ACEI/ARB use & $26(45.6 \%)$ & $45(63.4 \%)$ & 0.044 & $1(2.5 \%)$ & $71(77.2 \%)$ & $<0.001$ \\
\hline Statin use & $43(75.4 \%)$ & $59(83.1 \%)$ & 0.284 & $27(69.2 \%)$ & $77(83.7 \%)$ & 0.061 \\
\hline \multicolumn{7}{|l|}{ Sleep parameters } \\
\hline AHI & $8.8(3.2,17.3)$ & $12.9(7.3,26.7)$ & 0.013 & $6.1(1.7,14.5)$ & $14.6(8.0,28.4)$ & $<0.001$ \\
\hline ODI & $4.8(1.2,10.6)$ & $8.3(3.6,18.2)$ & 0.018 & $2.6(0.6,8.0)$ & $8.4(3.8,18.9)$ & $<0.001$ \\
\hline $\mathrm{AHI} \geq 5$ & $38(66.6 \%)$ & $58(81.7 \%)$ & 0.051 & $23(58.9 \%)$ & $76(82.6 \%)$ & 0.004 \\
\hline $\mathrm{AHI} \geq 15$ & $20(35.1 \%)$ & $32(45.1 \%)$ & 0.253 & $8(20.5 \%)$ & $45(48.9 \%)$ & 0.002 \\
\hline
\end{tabular}

Data are presented as mean (SD), median (IQR), or number (\%). ACEI: angiotensin-converting enzyme inhibitor; ARB: angiotensin receptor blocker; AHI: apnea hypopnea index; eGFR: estimated glomerular filtration rate; HbAlc: hemoglobin Alc; ODI: oxygen desaturation index.

TABLE 3: Mediation models of moderate-to-severe OSA $\rightarrow$ hypertension (HT) $\rightarrow$ any diabetes-related complications.

\begin{tabular}{lcccccc}
\hline Model & Factors & $B$ & SE & $t$ & $p$ & $95 \%$ CI \\
\hline \multirow{3}{*}{ Moderate-to-severe OSA $\rightarrow$ HT } & OSA & 1.074 & 0.488 & 2.201 & 0.028 & $0.118,2.031$ \\
& Age & 0.095 & 0.025 & 3.806 & 0.000 & $0.046,0.144$ \\
& BMI & 0.177 & 0.055 & 3.229 & 0.001 & $0.069,0.284$ \\
HT $\rightarrow$ any complications & Diabetes duration & -0.005 & 0.031 & -0.158 & 0.874 & $-0.065,0.055$ \\
& HT & 1.038 & 0.414 & 2.509 & 0.012 & $0.227,1.849$ \\
& OSA & 0.177 & 0.386 & 0.458 & 0.647 & $-0.580,0.934$ \\
\hline
\end{tabular}

$B$ : unstandardized coefficient; SE: standard error; $t=t$-test statistic.

than those without hypertension. More hypertensive patients also had moderate-to-severe OSA than those without hypertension $(p=0.002)$.

\subsection{Hypertension as a Mediator between OSA and Diabetes-}

Related Complications. Since those with any diabetesrelated complications had more severe OSA and were more likely to have hypertension and hypertension was strongly associated with OSA, we further explored if having hypertension could be a potential mediator between OSA and diabetes-related complications.

A mediation model was constructed by fitting OSA on hypertension, adjusting for covariables including age, BMI, and diabetes duration. This model indicated that T2DM patients with concurrent moderate-to-severe OSA were about 2.9 times more likely to have hypertension than those with mild or no OSA (odds ratio calculated as $e^{B}=e^{1.074}$; Table 3). A diabetes-related complication outcome model was then constructed. None of the covariables were significantly associated with diabetes-related complications; thus, only OSA and hypertension were included in this model. This indicated that patients with hypertension were 2.8 times more likely to have any diabetes-related complications than those without hypertension.
TABLE 4: Estimation of mediation effects in mediation pathway of moderate-to-severe OSA $\rightarrow$ hypertension $(\mathrm{HT}) \rightarrow$ any diabetesrelated complications.

\begin{tabular}{lccc}
\hline & Pathway & $\begin{array}{c}\text { Odds } \\
\text { ratio }\end{array}$ & $95 \%$ CI \\
\hline Indirect & $\begin{array}{c}\text { OSA } \rightarrow \text { HT } \rightarrow \text { any } \\
\text { complications }\end{array}$ & 3.049 & $1.026,25.775$ \\
Direct & OSA $\rightarrow$ any complications & 1.194 & $0.546,2.654$ \\
\hline
\end{tabular}

A bootstrap with 1000 replications was applied and revealed that T2DM patients with concurrent moderate-tosevere OSA would have an odds ratio of having any diabetes-related complications, mediated through having hypertension, of 3.05 (95\% CI: 1.03, 25.78) (Table 4 and Figure 1), while OSA had no direct effect on complications. This suggested that OSA was associated with hypertension, which in turn increased the risk of having diabetesrelated complications.

3.3. OSA Severity and eGFR. The association between OSA severity and eGFR was further explored. Table 5 demonstrates the results of univariate linear regression analysis 


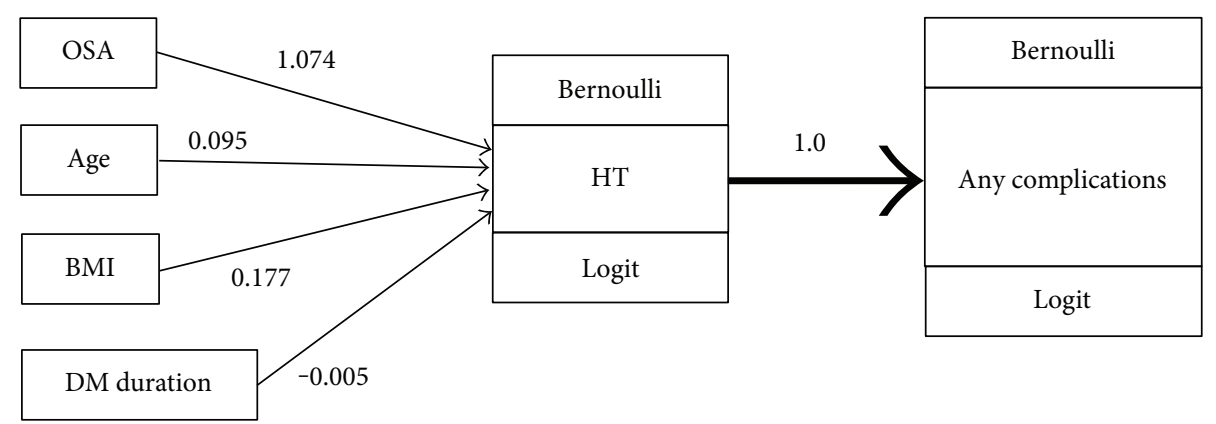

FIgURE 1: A mediation diagram of moderate-to-severe OSA $\rightarrow$ hypertension (HT) $\rightarrow$ any diabetes-related complications. Bernoulli distribution was regressed on OSA, adjusting for age, BMI, and DM duration, with logit link. An "any diabetes-related complications" model was constructed with similar distribution and link function.

TABLE 5: Univariate linear regression analysis to evaluate associations between baseline characteristics, sleep parameters, and Ln eGFR.

\begin{tabular}{lcc}
\hline Variables & $B$ & $p$ value \\
\hline Age & -0.016 & $<0.001$ \\
Sex & 0.093 & 0.114 \\
BMI & 0.011 & 0.064 \\
Ln diabetes duration & -0.087 & $<0.001$ \\
Ln HbA1c & 0.313 & 0.038 \\
Hypertension & -0.176 & 0.006 \\
ACEI/ARB use & -0.115 & 0.049 \\
Statin use & -0.133 & 0.066 \\
Ln AHI & -0.057 & 0.016 \\
Ln ODI & -0.050 & 0.014 \\
\hline
\end{tabular}

$B$ : unstandardized coefficient; ACEI: angiotensin-converting enzyme inhibitor; ARB: angiotensin receptor blocker; AHI: apnea hypopnea index; eGFR: estimated glomerular filtration rate; HbAlc: hemoglobin A1c; Ln: natural logarithm; ODI: oxygen desaturation index.

between demographic, glycemic, and sleep parameters and eGFR. Older age, longer diabetes duration, having hypertension, and statin or ACEI/ARB use were associated with lower eGFR. In addition, more severe OSA, as indicated by higher AHI and ODI, was significantly associated with lower eGFR.

To determine if OSA severity was an independent predictor of eGFR, a stepwise backward regression analysis, adjusting for age, BMI, diabetes duration, HbAlc, hypertension, ACEI/ARB use, and statin use, was performed (Table 6). This demonstrated that higher AHI was borderline associated with lower eGFR $(B=-0.037, p=0.063)$, while higher ODI was significantly associated with lower eGFR $(B=-0.036$, $p=0.041)$.

\section{Discussion}

In this study of T2DM patients, the results revealed that OSA was associated with diabetes-related complications, and this relationship was mediated by the presence of hypertension. The mediation analysis suggested that T2DM patients with moderate-to-severe OSA and hypertension were three times more likely to have diabetes-related complications compared
TABLE 6: Stepwise backward regression analysis to determine the independent predictors of Ln eGFR.

\begin{tabular}{lcccc}
\hline Variables & $B$ & $p$ value & $B$ & $p$ value \\
\hline Age & -0.015 & $<0.001$ & -0.015 & $<0.001$ \\
Ln AHI & -0.037 & 0.063 & - & - \\
Ln ODI & & & -0.036 & 0.041 \\
\hline
\end{tabular}

AHI: apnea hypopnea index; eGFR: estimated glomerular filtration rate; Ln: natural logarithm; ODI: oxygen desaturation index.

to those with no or mild OSA. In addition, the degree of intermittent hypoxia (ODI) was found to be independently associated with lower eGFR in this group of patients without ESRD, after adjusting for multiple confounders including hypertensive status. Collectively, these data support the role of OSA in diabetes-related complications and that hypertension plays at least a partial role in these relationships.

The data from this current study are in agreement with several previous studies demonstrating the effects of OSA on diabetic micro- and macrovascular complications. For example, a longitudinal study in 132 T2DM patients, followed for 4.9 years, revealed that sleep-disordered breathing was a predictor of incident coronary artery disease with a hazard ratio of 1.9 (95\% CI: 1.1, 3.3), as well as an increased risk for heart failure [29]. In the Sleep AHEAD study which included 305 T2DM patients, AHI was associated with a 2.57-fold increase in risk of having a history of stroke [30]. Similar associations were found for microvascular complications. While a systematic review of eight studies found no convincing evidence that OSA was associated with diabetic retinopathy [17], a more recent longitudinal study revealed that OSA was a risk factor for progression to advanced diabetic retinopathy (odds ratio [OR], 5.2; 95\% CI: 1.2, 23.0) [18]. A meta-analysis of five studies (880 patients) demonstrated an association between OSA and diabetic neuropathy (a pooled OR of 1.90), with a higher risk seen in those younger than 60 [31]. A more detailed assessment by skin biopsies from 52 T2DM patients revealed that higher AHI was associated with lower intraepidermal nerve fiber density, suggesting small fiber neuropathy [19]. The data from this current study suggested that hypertension plays an important role in the association between OSA and diabetes-related complications seen in this cohort. This is not surprising, as OSA is associated with hypertension, and CPAP treatment was 
shown to be beneficial in those with resistant hypertension [32]. Tighter blood pressure control in T2DM (144/82 versus $154 / 87 \mathrm{mmHg}$ ) was shown to reduce the risk of retinopathy and albuminuria [33], although lowering blood pressure to a target systolic of $<120 \mathrm{mmHg}$ did not further reduce the progression of retinopathy [34].

Only a few studies have explored the relationship between eGFR and OSA severity exclusively in T2DM. A crosssectional study in 90 participants by Leong et al. found that $\mathrm{AHI}$ and time spent under $\mathrm{O}_{2}$ saturation of $90 \%$ were negatively correlated with eGFR, suggesting the adverse effects of hypoxemia [35]. This was confirmed in a larger study of 880 hospitalized patients in China, which demonstrated a negative association between ODI and eGFR [36]. In addition, a cohort study with a follow-up of 2.5 years revealed that patients with T2DM and OSA had a faster decline in their eGFR than those without OSA, with baseline nitrosative stress being an independent predictor of eGFR decline [37]. The current results are in agreement with these previous findings and underscore the importance of intermittent hypoxia as ODI, but not AHI, related to lower eGFR. In addition, patients with ESRD were excluded from the current study, and a majority of the patients (89.3\%) had eGFR $\geq 60 \mathrm{~mL} /$ $\mathrm{min} / 1.73 \mathrm{~m}^{2}$; thus, the relationship between OSA and renal function exists even in the early stages of DKD.

Several potential mechanistic pathways could explain the link between OSA and diabetes-related complications. Intermittent hypoxemia leads to vasoconstriction and increased oxidative stress, such as alterations in nitric oxide synthase activity, resulting in endothelial dysfunction and microvascular impairment [20]. Increased sympathetic nervous system activity, increased endothelin-1 levels, and alterations in renin-angiotensin-aldosterone regulation could also contribute to elevated blood pressure [38], and the latter could mediate the relationship between OSA and DKD. Nighttime hypoxemia was found to lead to an increase in vascular endothelial growth factor (VEGF) [39], a hypoxia-sensitive glycoprotein stimulating neoangiogenesis, which has been shown to play a role in diabetic retinopathy [40]. The OSA-related increase of inflammatory markers and hypercoagulability are also likely factors $[20,41]$. In addition, OSA can impact glycemic control in T2DM, although this relationship was not found in the current cohort (data not shown). Increased advanced glycation end products and alterations in protein kinase $\mathrm{C}$ signaling are common pathways by which OSA and hyperglycemia contribute to microvascular complications $[42,43]$. Through these multiple pathways, OSA could exert adverse effects on diabetes-related complications.

These data suggested that CPAP could be beneficial in reducing diabetes-related complications. While there is evidence supporting the favorable effects on blood pressure in T2DM [44, 45], no randomized trials exploring the effects of CPAP on diabetes-related complications have been conducted. Thus, current data were mostly observational. In a small cohort of 47 patients, those who were compliant with CPAP therapy had a slower decline of their eGFR after 2.5 years [37]. In another study in 38 T2DM patients, those who were compliant with CPAP were less likely to progress to advanced retinopathy [18]. As CPAP treatment was shown to downregulate renin-angiotensin-aldosterone activity [46] and reduce VEGF levels [47] in those without diabetes, these could possibly be mechanistic pathways by which CPAP use is associated with reduced risk of DKD and retinopathy. No data are available with regard to neuropathy. Thus, further research in this area is warranted, as OSA is very prevalent in the T2DM population and measures are needed to reduce morbidity and mortality related to diabetic complications.

The strength of this study was a comprehensive assessment of diabetes-related complications by applying mediation analysis. The exclusion of patients with ESRD revealed the relationship between OSA and eGFR even in patients with relatively normal renal function. However, there are limitations. The cross-sectional design precludes an assumption of a causal relationship. Importantly, there could be differing relationships between OSA and diabetes-related complications at different blood pressure levels or circadian variations. There is evidence that OSA is associated with a nondipping pattern of nocturnal blood pressure [48, 49], which has been shown to be associated with albuminuria and reduced eGFR in T2DM [50], as well as increased allcause mortality [51]. Lack of comprehensive blood pressure measurements, especially in a longitudinal manner or with 24-hour monitoring, did not allow us to evaluate this possible relationship. Moreover, the compliance with antihypertensive therapies was not available. In this study, only moderate-to-severe OSA was used in the mediation analysis, as $\mathrm{AHI}$ or $\mathrm{AHI} \geq 5$ was not found to be associated with diabetes-related complications after adjusting for hypertension (data not shown). This, however, was consistent with evidence of a dose-response relationship between OSA severity and adverse health outcomes [23]. In addition, standard polysomnography was not used in this study, and WatchPAT could not differentiate central from obstructive events. However, we excluded those with risk factors for central apnea, as described above. Because of this, more severe diabetesrelated complications such as stroke and heart failure were not evaluated in this study, even though these were reported to be associated with OSA in the general population $[52,53]$. Lastly, the overall sample size of 131 may be relatively small to detect associations in few causal pathways of mediation analysis. As for estimations of post hoc power of the test, the powers were $73 \%, 93.3 \%$, and $52.1 \%$ for OSA $\rightarrow$ hypertension, hypertension $\rightarrow$ diabetes-related complications, and OSA $\rightarrow$ diabetes-related complications, respectively.

In conclusion, OSA, especially moderate-to-severe degree, was associated with diabetes-related complications in T2DM. This relationship was mediated by hypertensive state. In addition, more severe intermittent hypoxemia was related to lower eGFR, independently of hypertension, in this patient group with relatively normal renal function. Further studies exploring the effects of CPAP on diabetes-related complications are warranted.

\section{Conflicts of Interest}

Sirimon Reutrakul received grant support from Merck Sharp and Dohme, research equipment support from ResMed, and 
speaker honoraria from Sanofi, Novo Nordisk, and Medtronic. All other authors reported no conflict of interest.

\section{Authors' Contributions}

Nantaporn Siwasaranond researched and analyzed the data and wrote and edited the manuscript. Hataikarn Nimitphong, Areesa Manodpitipong, and Sunee Saetung researched the data and reviewed/edited the manuscript. Naricha Chirakalwasan participated in study planning and reviewed/edited the manuscript. Ammarin Thakkinstian analyzed and interpreted the data and reviewed/edited the manuscript. Sirimon Reutrakul planned the study, researched and analyzed the data, and wrote/edited the manuscript.

\section{Acknowledgments}

This study was supported by a grant from the Faculty of Medicine Ramathibodi Hospital, Mahidol University, Bangkok, Thailand, and the Endocrine Society of Thailand.

\section{References}

[1] S. Reutrakul and B. Mokhlesi, "Obstructive sleep apnea and diabetes: a state of the art review," Chest, vol. 152, no. 5, pp. 1070-1086, 2017.

[2] M. Louis and N. M. Punjabi, "Effects of acute intermittent hypoxia on glucose metabolism in awake healthy volunteers," Journal of Applied Physiology, vol. 106, no. 5, pp. 1538-1544, 2009.

[3] E. Tasali, R. Leproult, D. A. Ehrmann, and E. Van Cauter, "Slow-wave sleep and the risk of type 2 diabetes in humans," Proceedings of the National Academy of Sciences, vol. 105, no. 3, pp. 1044-1049, 2008.

[4] T. Young, J. Skatrud, and P. E. Peppard, "Risk factors for obstructive sleep apnea in adults," Journal of the American Medical Association, vol. 291, no. 16, pp. 2013-2016, 2004.

[5] L. S. Aboussouan, "Sleep-disordered breathing in neuromuscular disease," American Journal of Respiratory and Critical Care Medicine, vol. 191, no. 9, pp. 979-989, 2015.

[6] M. A. Gupta and F. C. Simpson, "Obstructive sleep apnea and psychiatric disorders: a systematic review," Journal of Clinical Sleep Medicine, vol. 11, no. 2, pp. 165-175, 2015.

[7] N. M. Punjabi, "The epidemiology of adult obstructive sleep apnea," Proceedings of the American Thoracic Society, vol. 5, no. 2, pp. 136-143, 2008.

[8] K. Mahmood, N. Akhter, K. Eldeirawi et al., "Prevalence of type 2 diabetes in patients with obstructive sleep apnea in a multi-ethnic sample," Journal of Clinical Sleep Medicine, vol. 5, no. 3, pp. 215-221, 2009.

[9] G. D. Foster, M. H. Sanders, R. Millman et al., "Obstructive sleep apnea among obese patients with type 2 diabetes," Diabetes Care, vol. 32, no. 6, pp. 1017-1019, 2009.

[10] M. P. Hermans, S. A. Ahn, Y. P. Mahadeb, and M. F. Rousseau, "Sleep apnoea syndrome and 10-year cardiovascular risk in females with type 2 diabetes: relationship with insulin secretion and insulin resistance," Diabetes/Metabolism Research and Reviews, vol. 29, no. 3, pp. 227-234, 2013.

[11] R. S. Aronsohn, H. Whitmore, E. Van Cauter, and E. Tasali, "Impact of untreated obstructive sleep apnea on glucose control in type 2 diabetes," American Journal of Respiratory and Critical Care Medicine, vol. 181, no. 5, pp. 507-513, 2010.

[12] E. Martinez-Ceron, B. Barquiel, A. M. Bezos et al., "Effect of continuous positive airway pressure on glycemic control in patients with obstructive sleep apnea and type 2 diabetes. A randomized clinical trial," American Journal of Respiratory and Critical Care Medicine, vol. 194, no. 4, pp. 476485, 2016.

[13] J. E. Shaw, N. M. Punjabi, M. T. Naughton et al., "The effect of treatment of obstructive sleep apnea on glycemic control in type 2 diabetes," American Journal of Respiratory and Critical Care Medicine, vol. 194, no. 4, pp. 486-492, 2016.

[14] UK Prospective Diabetes Study Group, "Intensive bloodglucose control with sulphonylureas or insulin compared with conventional treatment and risk of complications in patients with type 2 diabetes (UKPDS 33)," The Lancet, vol. 352, no. 9131, pp. 837-853, 1998.

[15] P. Gaede, P. Vedel, N. Larsen, G. V. Jensen, H. H. Parving, and O. Pedersen, "Multifactorial intervention and cardiovascular disease in patients with type 2 diabetes," New England Journal of Medicine, vol. 348, no. 5, pp. 383-393, 2003.

[16] W. B. Leong, F. Jadhakhan, S. Taheri, G. N. Thomas, and P. Adab, "The association between obstructive sleep apnea on diabetic kidney disease: a systematic review and meta-analysis," Sleep, vol. 39, no. 2, pp. 301-308, 2016.

[17] W. B. Leong, F. Jadhakhan, S. Taheri, Y. F. Chen, P. Adab, and G. N. Thomas, "Effect of obstructive sleep apnoea on diabetic retinopathy and maculopathy: a systematic review and meta-analysis," Diabetic Medicine, vol. 33, no. 2, pp. 158$168,2016$.

[18] Q. A. Altaf, P. Dodson, A. Ali et al., “Obstructive sleep apnoea and retinopathy in patients with type 2 diabetes: a longitudinal study," American Journal of Respiratory and Critical Care Medicine, vol. 196, no. 7, pp. 892-900, 2017.

[19] Q. A. Altaf, A. Ali, M. K. Piya, N. T. Raymond, and A. A. Tahrani, "The relationship between obstructive sleep apnea and intra-epidermal nerve fiber density, PARP activation and foot ulceration in patients with type 2 diabetes," Journal of Diabetes and its Complications, vol. 30, no. 7, pp. 13151320, 2016.

[20] L. Lavie, "Oxidative stress in obstructive sleep apnea and intermittent hypoxia-revisited-the bad ugly and good: implications to the heart and brain," Sleep Medicine Reviews, vol. 20, pp. 27-45, 2015.

[21] N. F. Turek, A. C. Ricardo, and J. P. Lash, “Sleep disturbances as nontraditional risk factors for development and progression of CKD: review of the evidence," American Journal of Kidney Diseases, vol. 60, no. 5, pp. 823-833, 2012.

[22] O. Friedman and A. G. Logan, "The price of obstructive sleep apnea-hypopnea: hypertension and other ill effects," American Journal of Hypertension, vol. 22, no. 5, pp. 474-483, 2009.

[23] A. Cai, L. Wang, and Y. Zhou, "Hypertension and obstructive sleep apnea," Hypertension Research, vol. 39, no. 6, pp. 391395, 2016.

[24] A. I. Adler, I. M. Stratton, H. A. Neil et al., "Association of systolic blood pressure with macrovascular and microvascular complications of type 2 diabetes (UKPDS 36): prospective observational study," BMJ, vol. 321, no. 7258, pp. 412-419, 2000.

[25] A. Manodpitipong, S. Saetung, H. Nimitphong et al., "Nightshift work is associated with poorer glycaemic control in 
patients with type 2 diabetes," Journal of Sleep Research, vol. 26, no. 6, pp. 764-772, 2017.

[26] N. Siwasaranond, H. Nimitphong, S. Saetung, N. Chirakalwasan, B. Ongphiphadhanakul, and S. Reutrakul, "Shorter sleep duration is associated with poorer glycemic control in type 2 diabetes patients with untreated sleepdisordered breathing," Sleep \& Breathing, vol. 20, no. 2, pp. 569-574, 2016.

[27] M. Yuceege, H. Firat, A. Demir, and S. Ardic, "Reliability of the Watch-PAT 200 in detecting sleep apnea in highway bus drivers," Journal of Clinical Sleep Medicine, vol. 9, no. 4, pp. 339-344, 2013.

[28] D. P. MacKinnon, A. J. Fairchild, and M. S. Fritz, "Mediation analysis," Annual Review of Psychology, vol. 58, no. 1, pp. 593-614, 2007.

[29] S. Seicean, K. P. Strohl, A. Seicean, C. Gibby, and T. H. Marwick, "Sleep disordered breathing as a risk of cardiac events in subjects with diabetes mellitus and normal exercise echocardiographic findings," The American Journal of Cardiology, vol. 111, no. 8, pp. 1214-1220, 2013.

[30] T. B. Rice, G. D. Foster, M. H. Sanders et al., "The relationship between obstructive sleep apnea and self-reported stroke or coronary heart disease in overweight and obese adults with type 2 diabetes mellitus," Sleep, vol. 35, no. 9, pp. 1293-1298, 2012.

[31] K. Fujihara, S. Kodama, C. Horikawa et al., "The relationship between diabetic neuropathy and sleep apnea syndrome: a meta-analysis," Sleep Disorders, vol. 2013, Article ID 150371, 7 pages, 2013.

[32] I. H. Iftikhar, C. W. Valentine, L. R. Bittencourt et al., "Effects of continuous positive airway pressure on blood pressure in patients with resistant hypertension and obstructive sleep apnea: a meta-analysis," Journal of Hypertension, vol. 32, no. 12, pp. 2341-2350, 2014.

[33] UK Prospective Diabetes Study Group, “Tight blood pressure control and risk of macrovascular and microvascular complications in type 2 diabetes: UKPDS 38," BMJ, vol. 317, no. 7160, pp. 703-713, 1998.

[34] ACCORD Study Group; ACCORD Eye Study Group, E. Y. Chew, W. T. Ambrosius et al., "Effects of medical therapies on retinopathy progression in type 2 diabetes," New England Journal of Medicine, vol. 363, no. 3, pp. 233-244, 2010.

[35] W. B. Leong, M. Nolen, G. N. Thomas, P. Adab, D. Banerjee, and S. Taheri, "The impact of hypoxemia on nephropathy in extremely obese patients with type 2 diabetes mellitus," Journal of Clinical Sleep Medicine, vol. 10, no. 7, pp. 773778, 2014.

[36] R. Zhang, P. Zhang, F. Zhao, X. Han, and L. Ji, “Association of diabetic microvascular complications and parameters of obstructive sleep apnea in patients with type 2 diabetes," Diabetes Technology \& Therapeutics, vol. 18, no. 7, pp. 415-420, 2016.

[37] A. A. Tahrani, A. Ali, N. T. Raymond et al., "Obstructive sleep apnea and diabetic nephropathy: a cohort study," Diabetes Care, vol. 36, no. 11, pp. 3718-3725, 2013.

[38] B. G. Phillips, K. Narkiewicz, C. A. Pesek, W. G. Haynes, M. E. Dyken, and V. K. Somers, "Effects of obstructive sleep apnea on endothelin-1 and blood pressure," Journal of Hypertension, vol. 17, no. 1, pp. 61-66, 1999.

[39] R. Schulz, C. Hummel, S. Heinemann, W. Seeger, and F. Grimminger, "Serum levels of vascular endothelial growth factor are elevated in patients with obstructive sleep apnea and severe nighttime hypoxia," American Journal of Respiratory and Critical Care Medicine, vol. 165, no. 1, pp. 67-70, 2002.

[40] C. C. Wykoff, "Impact of intravitreal pharmacotherapies including antivascular endothelial growth factor and corticosteroid agents on diabetic retinopathy," Current Opinion in Ophthalmology, vol. 28, no. 3, pp. 213-218, 2017.

[41] C. Rangemark, J. A. Hedner, J. T. Carlson, G. Gleerup, and K. Winther, "Platelet function and fibrinolytic activity in hypertensive and normotensive sleep apnea patients," Sleep, vol. 18, no. 3, pp. 188-194, 1995.

[42] B. R. Webster, J. M. Osmond, D. A. Paredes et al., "Phosphoinositide-dependent kinase- 1 and protein kinase $\mathrm{C} \delta$ contribute to endothelin-1 constriction and elevated blood pressure in intermittent hypoxia," The Journal of Pharmacology and Experimental Therapeutics, vol. 344, no. 1, pp. 68-76, 2013.

[43] J. C. Lam, K. C. Tan, A. Y. Lai, D. C. Lam, and M. S. Ip, "Increased serum levels of advanced glycation end-products is associated with severity of sleep disordered breathing but not insulin sensitivity in non-diabetic men with obstructive sleep apnoea," Sleep Medicine, vol. 13, no. 1, pp. 15-20, 2012.

[44] J. C. M. Lam, A. Y. K. Lai, T. C. C. Tam, M. M. A. Yuen, K. S. L. Lam, and M. S. M. Ip, "CPAP therapy for patients with sleep apnea and type 2 diabetes mellitus improves control of blood pressure," Sleep \& Breathing, vol. 21, no. 2, pp. 377-386, 2017.

[45] P. C. Myhill, W. A. Davis, K. E. Peters, S. A. Chubb, D. Hillman, and T. M. Davis, "Effect of continuous positive airway pressure therapy on cardiovascular risk factors in patients with type 2 diabetes and obstructive sleep apnea," Journal of Pharmacology and Experimental Therapeutics, vol. 97, no. 11, pp. 4212-4218, 2012.

[46] D. D. Nicholl, P. J. Hanly, M. J. Poulin et al., "Evaluation of continuous positive airway pressure therapy on reninangiotensin system activity in obstructive sleep apnea," American Journal of Respiratory and Critical Care Medicine, vol. 190, no. 5, pp. 572-580, 2014.

[47] K. Archontogeorgis, E. Nena, N. Papanas et al., "Serum levels of vascular endothelial growth factor and insulin-like growth factor binding protein-3 in obstructive sleep apnea patients: effect of continuous positive airway pressure treatment," The Open Cardiovascular Medicine Journal, vol. 9, no. 1, pp. 133138, 2015.

[48] Y. Ma, S. Sun, C. K. Peng, Y. Fang, and R. J. Thomas, “Ambulatory blood pressure monitoring in chinese patients with obstructive sleep apnea," Journal of Clinical Sleep Medicine, vol. 13, no. 03, pp. 433-439, 2017.

[49] B. Mokhlesi, E. W. Hagen, L. A. Finn, K. M. Hla, J. R. Carter, and P. E. Peppard, "Obstructive sleep apnoea during REM sleep and incident non-dipping of nocturnal blood pressure: a longitudinal analysis of the Wisconsin sleep cohort," Thorax, vol. 70, no. 11, pp. 1062-1069, 2015.

[50] P. E. Jennersjo, M. Wijkman, A. B. Wirehn et al., "Circadian blood pressure variation in patients with type 2 diabetes-relationship to macro- and microvascular subclinical organ damage," Primary Care Diabetes, vol. 5, no. 3, pp. 167$173,2011$.

[51] D. J. Brotman, M. B. Davidson, M. Boumitri, and D. G. Vidt, "Impaired diurnal blood pressure variation and all-cause mortality," American journal of hypertension., vol. 21, no. 1, pp. 92-97, 2008. 
[52] K. G. Johnson and D. C. Johnson, "Frequency of sleep apnea in stroke and TIA patients: a meta-analysis," Journal of Clinical Sleep Medicine, vol. 6, no. 2, pp. 131-137, 2010.

[53] M. Arzt, O. Oldenburg, A. Graml et al., "Phenotyping of sleep-disordered breathing in patients with chronic heart failure with reduced ejection fraction-the SchlaHF registry," Journal of the American Heart Association, vol. 6, no. 12, article e005899, 2017. 


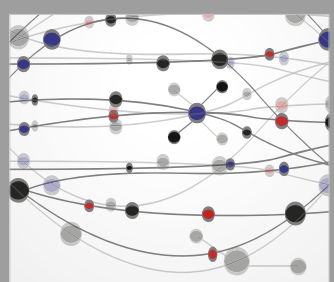

The Scientific World Journal
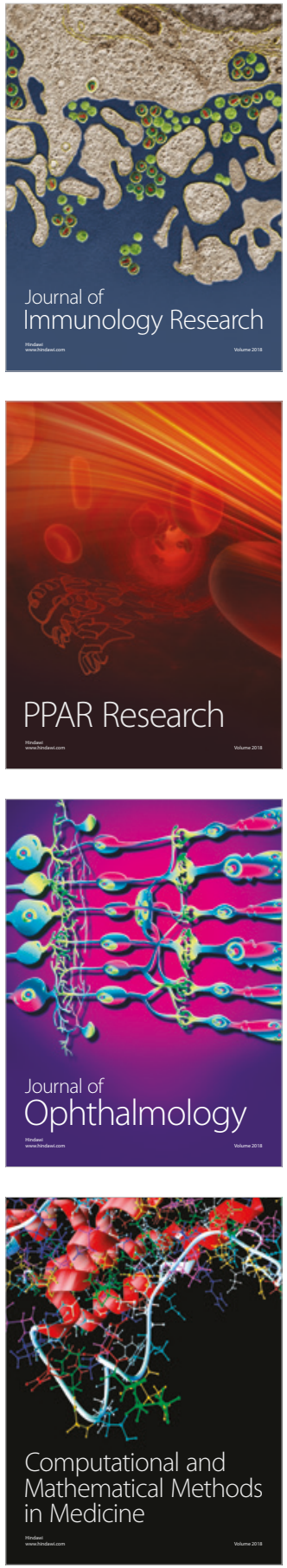

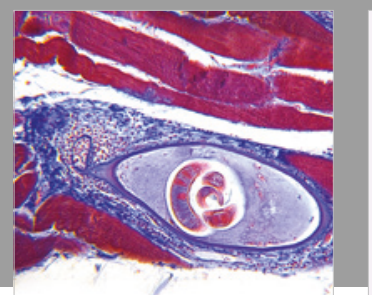

Gastroenterology Research and Practice

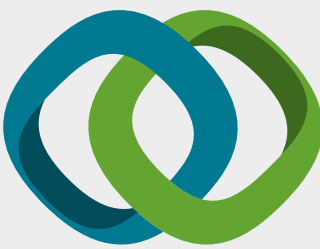

\section{Hindawi}

Submit your manuscripts at

www.hindawi.com
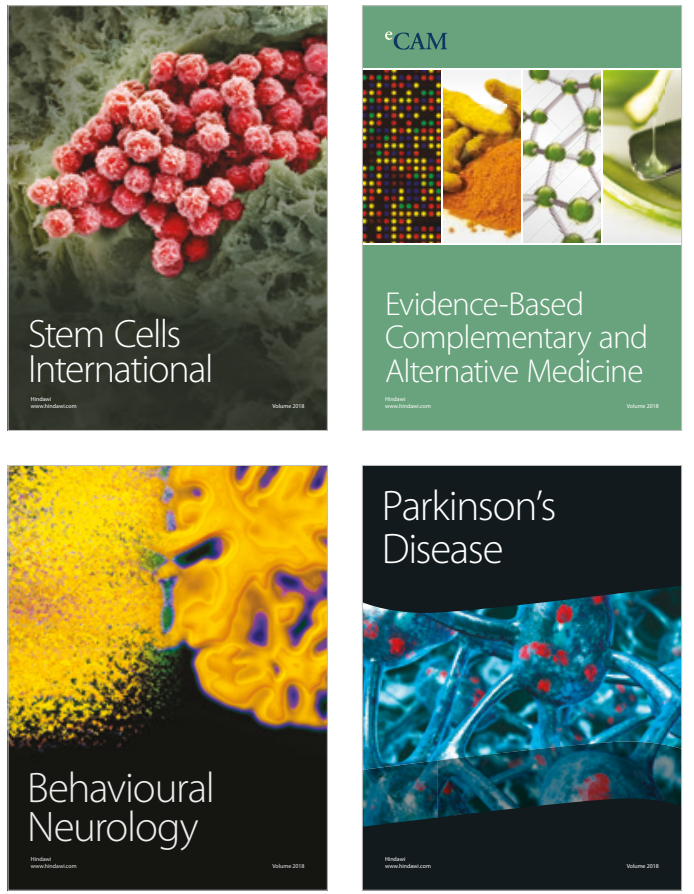

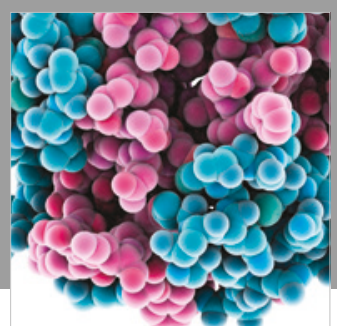

ournal of

Diabetes Research

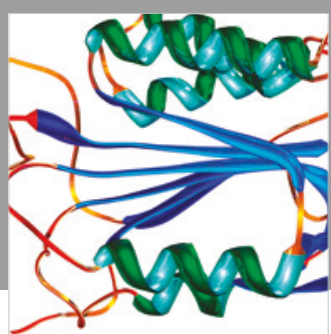

Disease Markers
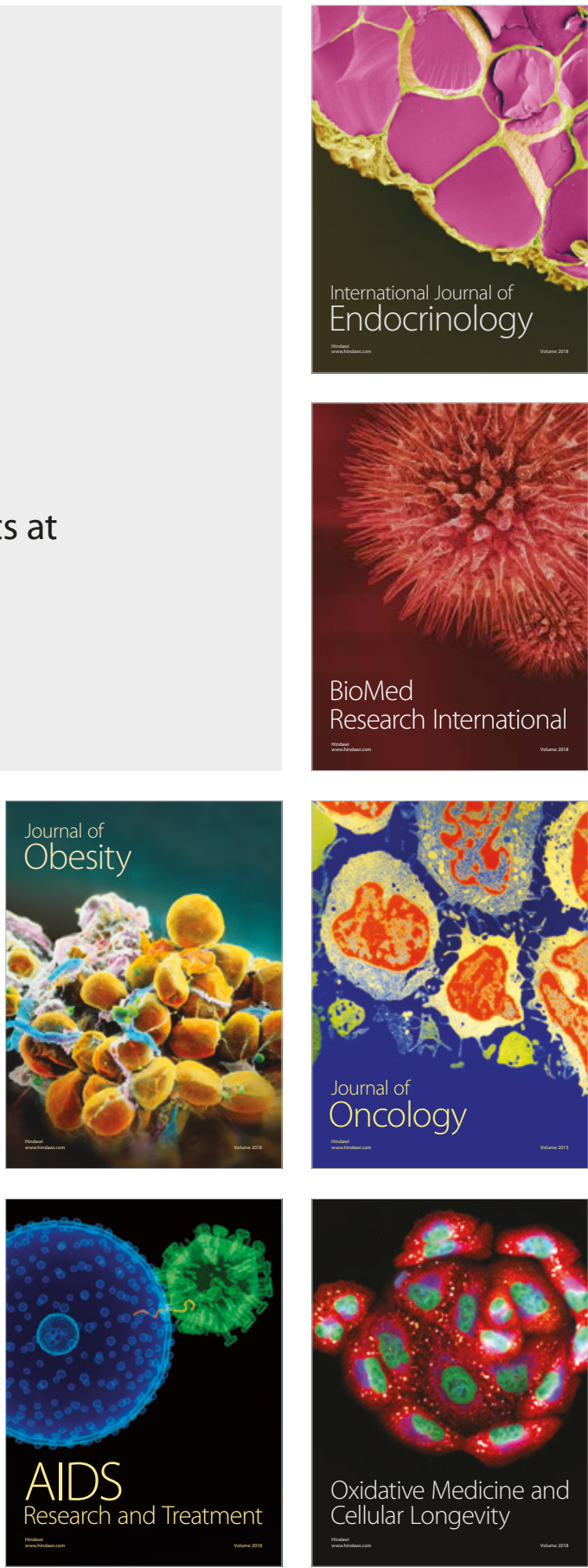